\title{
Análise geométrica do estudo das posições relativas entre reta e plano com o Geogebra
}

\author{
Geometric analysis of the study of relative positions between straight and plane \\ with Geogebra
}

Rildenir Ribeiro Silva ril.ifmatematico@gmail.com

Julia Anjos Igreja jubsigreja@gmail.com

Maxwell Pereira de Oliveira mto8005@hotmail.com

Polianna Geysa Rodrigues polianna.rodrigues@,outlook.com

Sandoval Alexandre Junior sjr.juniormaster@hotmail.com

Este trabalho teve como ponto de partida viabilizar uma nova forma de se analisar e representar os resultados analíticos encontrados na resolução de problemas do estudo das posições relativas entre retas e planos, assunto este abordado em Geometria Analítica nos cursos de graduação das áreas de exatas como Matemática, Física, Química, Engenharias, Sistemas de Informação, etc. Nosso objetivo é dar um enfoque geométrico no desenvolvimento da resolução dos problemas de posição relativa entre reta e reta, reta e plano e plano e plano, onde este assunto é visto em muitas práticas pedagógicas apenas de maneira superficial, com um tratado teórico. As análises, com esta nova metodologia de ensino foi implementada durante as aulas de Cálculo Vetorial e Geometria Analítica em laboratórios de informática do IFMA e da Faculdade FACAM, apresentando os conteúdos teóricos trabalhados em sala de aula com a aplicação do software Geogebra 5.0, para a verificação e validação dos resultados. Os estudos analíticos estão fundamentados em Boulos e Camargo (2005), principal referencial teórico adotado nos cursos de ensino superior das áreas de exatas. Utilizamos o software dinâmico, como uma metodologia alternativa, para auxiliar na complementação das atividades teóricas, almejando um pleno desenvolvimento deste conteúdo de nível superior, uma vez que tal dinamização do conhecimento pode ser inserida em diversas outras aplicações com este recurso, proporcionando novas possibilidades para o ensino e aprendizagem da Matemática e Geometria.

Palavras-chave: Ensino aprendizagem; Software dinâmico Geogebra; Geometria analítica.

\footnotetext{
Abstract

This work had as a starting point enabling a new way to analyze and represent the analytical results found in resolving positions of study problems related between lines and planes, issue this addressed in Analytic Geometry in undergraduate courses of the exact areas like Mathematics, Physics, Chemistry, Engineering, Information Systems,
} 
etc. Our goal is to give a geometrical approach in the development of solving the problems of relative position between straight, straight, straight and plan and plan and plan where this issue is see in many pedagogical practices only superficially, with a theoretical treatise. Analyses with this new teaching methodology was implemented during the Vector Calculus class and Analytic Geometry at IFMA's computer labs and have recourse to presenting the theoretical concepts learned in the classroom with the application of Geogebra 5.0 software for the verification and validation of the results. Analytical studies are based on Paul Boulos and Ivan de Camargo, the main theoretical framework adopted in higher education courses in the exact areas. We use the dynamic software, as an alternative methodology to assist in the completion of theoretical activities, aiming for full development of this top-level content, since such dynamic knowledge can be inserted in several other applications with this feature, providing new possibilities for teaching and learning of mathematics and geometry.

Keywords: Teaching and learning; Dynamic Geogebra software; Analytic geometry.

\section{Introdução}

Pesquisas recentes sobre o ensino e aprendizagem da matemática revelam que esta disciplina é um dos principais componentes curriculares com grande déficit de desempenho nas escolas de muitos países. As dificuldades podem começar no ensino básico, onde possivelmente irá se perdurar ao longo da vida acadêmica do aluno. Porém, paralelamente a estes problemas, está o desenvolvimento tecnológico das grandes mídias, meios de comunicação e informação, os quais, não deve estar dissociado das práticas docente durante o ensino. Os avanços tecnológicos devem ser instigados dentro do ambiente escolar acadêmico no sentido de viabilizar o ensino-aprendizagem, estreitando o caminho para o ensinar e aprender, em que professor e aluno são os protagonistas do grande desafio que é a aquisição do conhecimento de forma plena, significativa e aprimorada cada vez mais pelos aparatos da tecnologia, disponíveis para dinamizar o ensino em sala de aula facilitando o aprendizado de determinados conteúdos, fomentando à pesquisa científica de discentes e docentes.

Diante do advento da tecnologia e, sobretudo, nas salas de aula ao longo de décadas, a utilização das tecnologias da Informação e Comunicação no ensino da matemática vem pontualmente, se firmando como uma das áreas mais ativas e relevantes no aprimoramento de novas abordagens e ferramentas de ensino. A disponibilidade de inúmeros recursos como internet e softwares dinâmicos trabalhados de forma planejada e orientada, vem sendo de grande relevância para o surgimento de novas capacidades, abrindo um leque de possibilidades didáticas, modificando inclusive as relações entre professor e aluno no processo de ensinar e aprender. Segundo 
D’Ambrósio e Barros (1990), essas mudanças causam grandes impactos na sociedade, gerando reflexos conceituais e curriculares na educação básica e na educação superior.

Para fundamentar nossa pesquisa sobre esses avanços que intercederam à matemática, encontramos evidências nos Parâmetros Curriculares Nacionais (PCN’s), em que:

Esse impacto da tecnologia, cujo instrumento mais relevante é hoje o computador, exigirá do ensino de Matemática um redirecionamento sob uma perspectiva curricular que favoreça o desenvolvimento de habilidades e procedimentos com os quais o indivíduo possa se reconhecer e se orientar nesse mundo do conhecimento em constante movimento. (PCN's, 2000, p. 41)

O espaço da sala de aula, sem dúvida, não poderia estar de fora dessas transformações que ocorrem no mundo moderno com surgimentos de novas tecnologias. Com o passar dos anos, propostas voltadas para a utilização de Tecnologias de Informação e Comunicação (TICs) estão cada vez mais presentes em várias instâncias dentro da educação, seja por meio de políticas públicas ou até mesmo por desenvolvimento de pesquisa no mundo acadêmico e os Parâmetros Curriculares Nacionais (PCNs) são bem claros ao apontar com relevância o ensino da matemática com utilização de tecnologias. Diante do novo cenário e realidades distintas encontradas nas salas de aula, por meios das TICs, devemos utilizar as novas tecnologias:

\begin{abstract}
De comum acordo com o ensino desenvolvido, a avaliação deve dar informação sobre o conhecimento e compreensão de conceitos e procedimentos; a capacidade para aplicar conhecimentos na resolução de problemas do cotidiano; a capacidade para utilizar as linguagens das Ciências, da Matemática e suas Tecnologias para comunicar idéias; e as habilidades de pensamento como analisar, generalizar, inferir. (BRASIL, 2000, p.54).
\end{abstract}

Ainda sobre estes avanços e transformações, verificamos o que diz Borba (1999):

\footnotetext{
A introdução das novas tecnologias - computadores, calculadoras gráficas e interfaces que se modificam a cada dia - tem levantado diversas questões. Dentre elas destaco as preocupações relativas às mudanças curriculares, às novas dinâmicas da sala de aula, ao "novo" papel do professor e ao papel do computador nesta sala de aula. (BORBA, 1999, p. 285)
}

No que diz respeito à eficácia da utilização de ferramentas computacionais no ensino da matemática, segundo as concepções de Ponte e Oliveira (2003), acreditam que elas possam trazer novas perspectivas no ensino da Matemática de modo profundamente inovador, reforçando o papel da linguagem gráfica e de novas formas de representação e relativizando a importância do cálculo e da manipulação simbólica”. Dessa forma, as 
atividades mediadas pelo uso de softwares permitirão ao professor explorar as distintas formas de representar um mesmo problema sendo de forma gráfica, algébrica ou por meio de tabelas.

Sendo nosso ponto de partida como campo de pesquisa, a Geometria Analítica é a área que mais tem sido beneficiada pelas transformações com a utilização das tecnologias, principalmente, no desenvolvimento de softwares específicos voltados para o seu processo de ensino e aprendizagem. Conforme diz Zullato (2002, p. 20) são frequentemente utilizados no ensino de Geometria e permitem trabalhar com Geometria Euclidiana Plana, Geometria Não-Euclidiana e Geometria Analítica, onde o mesmo ainda afirma que os softwares são utilizados com a intenção de mostrar as propriedades que estão sendo estudadas com o intuito de realizar a verificação e visualização de propriedades.

\section{Material e Métodos}

O ensino do Cálculo Vetorial e Geometria Analítica, especificamente, na abordagem das posições relativas entre reta e plano, a partir da utilização do software dinâmico GeoGebra, pode favorecer a construção de significados em Matemática a partir da representação de conceitos, estudos de propriedades intrínsecas às construções realizadas, bem como explorar, a partir da visualização, das formas algébrica e geométrica desses conceitos encontrados nos livros acadêmicos segundo Paulo Boulos e Ivan de Camargo. Essa forma dinâmica de aprendizado proposta pelo GeoGebra pode favorecer à interação e estreitar os caminhos entre aluno e as novas ferramentas computacionais de aprendizagem.

O GeoGebra é um software matemático livre que reúne geometria, álgebra e cálculo. Ele foi desenvolvido por Markus Hohenwarter da Universidade de Salzburg para educação matemática nas escolas. Por um lado, o GeoGebra é um sistema de geometria dinâmica, permitindo realizar construções tanto com pontos, vetores, segmentos, retas, como com funções que podem se modificar posteriormente de forma dinâmica.

Nesse trabalho implementamos em nossas atividades o Geogebra 5.0, o qual o mesmo apresenta uma plataforma para análise em duas e três dimensões, conforme mostra a figura 1 : 
Figura 1: Janela principal do Geogebra 5.0 gerada pelo autor.

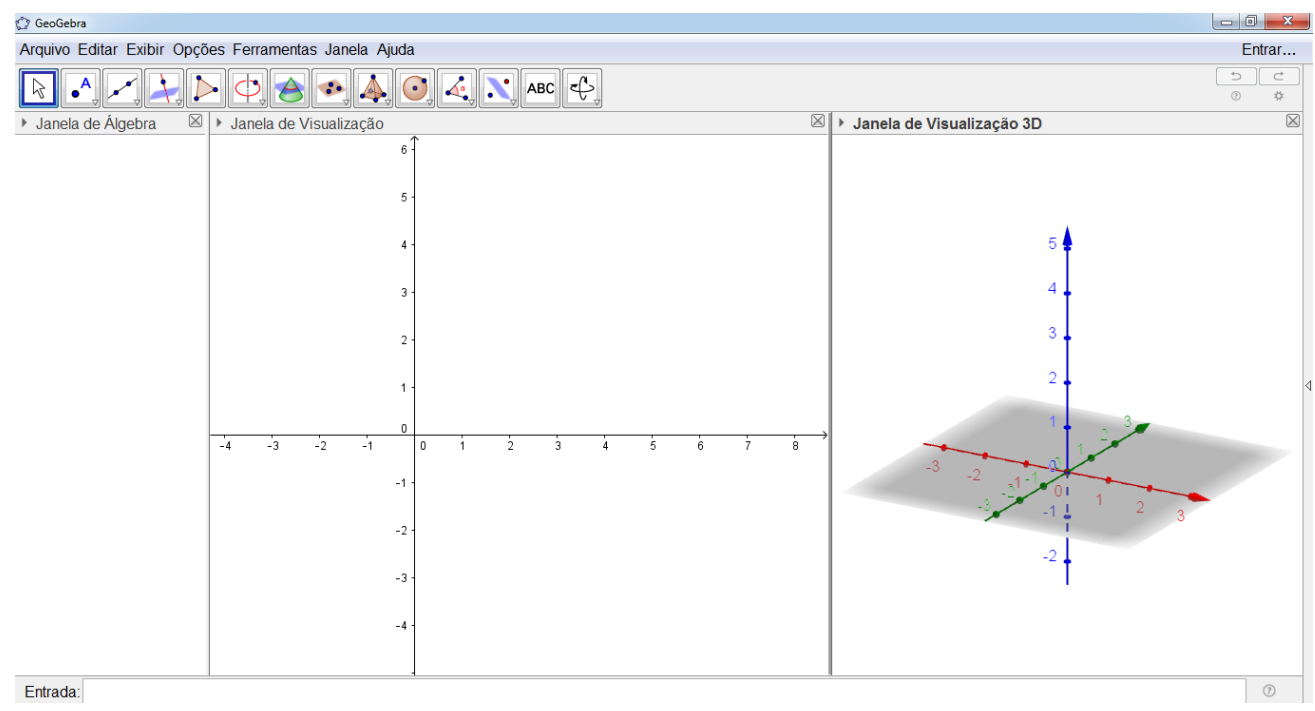

A partir do Geogebra 5.0, propomos como atividade exploratória de alguns exercícios do livro acadêmico: Geometria Analítica: Um tratamento Vetorial, do Paulo Boulos. As atividades são para verificar de forma gráfica, às propriedades que asseguram os conceitos sobre às posições relativas entre retas e planos estudadas no capítulo 16. Com base nos autores, foram preparadas 3 atividades que serão analisados o comportamento gráfico entre Reta e Reta, Reta e Pano e Plano e Plano, verificando às condições de forma geométrica.

\section{Resultados e Discussão}

As simulações e verificações foram realizadas pelos alunos de graduação do curso de Matemática da faculdade do Maranhão FACAM de maneira participativa e cooperativa com alunos do IFMA em laboratório de computação. A motivação para esta pesquisa, partiu da inquietação dos autores desta pesquisa, pelo fato de os problemas terem uma simples resolução, sem nenhum enfoque geométrico desses elementos. As investigações tiveram como ponto de partida, algumas atividades do livro já mencionado, em que propomos através de uma análise dinâmica, resolver os problemas propostos, proporcionando uma nova interpretação dos resultados analíticos obtidos nesta referência.

Atividade 1 - Reta e Reta 
Nesta atividade, iremos verificar à posição relativa entre duas retas $\boldsymbol{r}$ e $\boldsymbol{s}$. De acordo com (BOULOS e CAMARGO 2005, P.170), para tal verificação, devemos fixar um sistema de coordenadas no espaço e designar por $\overrightarrow{\boldsymbol{u}}=(\boldsymbol{a}, \boldsymbol{b}, \boldsymbol{c})$ um vetor diretor de $\boldsymbol{r}, \overrightarrow{\boldsymbol{v}}=(\boldsymbol{m}, \boldsymbol{n}, \boldsymbol{p})$ um vetor diretor de $\boldsymbol{s}$, por $\boldsymbol{A}=\left(\boldsymbol{x}_{\mathbf{1}}, \boldsymbol{y}_{\mathbf{1}}, \boldsymbol{z}_{\mathbf{1}}\right)$, um ponto qualquer de $\boldsymbol{r}$ e por $\boldsymbol{B}=\left(\boldsymbol{x}_{2}, \boldsymbol{y}_{2}, \boldsymbol{z}_{2}\right)$, um ponto qualquer de $\boldsymbol{s}$. Analisemos a $\mathbf{2}^{\mathbf{a}}$ questão: Estude a posição relativa entre as retas:

$r: X=(1,2,3)+\alpha(0,1,3), \quad \operatorname{com}(\alpha \in I R)$ e $s: X=(1,3,6)+\beta(0,2,6), \quad$ com $(\beta \in I R)$.

Para inserir os elementos geométricos no software, podemos utilizar a aba mostrada na figura 2 que segue:

Figura 2: Aba das principais ferramentas. Fonte Geogebra 5.0.

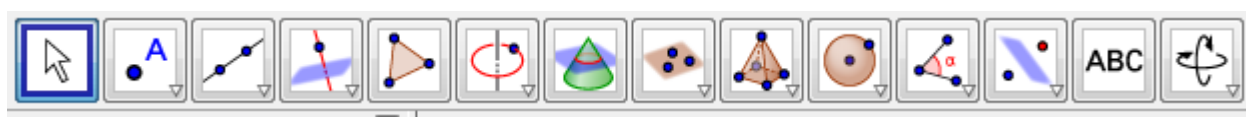

ou, então, no campo de entrada do Geogebra, digitar os parâmetros para cada reta dada, os pontos $A=(1,2,3)$ e $B=(1,3,6)$ e os respectivos vetores diretores das retas $r$ e $s$, $\vec{u}=(0,1,3)$ e $\vec{v}=(0,2,6)$. Para identificar as retas $r$ e $s$, digitamos no CAMPO DE ENTRADA o comando $\operatorname{reta}(A, u)$, isto é, queremos identificar a reta $r$ que passa pelo ponto $A$ e possui vetor diretor $u$, da mesma forma, para identificar a reta $s$ que passa pelo ponto $B$ e tem como vetor diretor $v$ digitamos $\operatorname{reta}(B, v)$. Os resultados da Atividade 1, ilustrados no gráfico 1 abaixo, mostram que, como os vetores são proporcionais, isto é, $\vec{v}=2 \vec{u}$, logo, $r$ e $s$ são paralelas, $r / / s$.

Figura 3: Interpretação geométrica da Atividade 1.

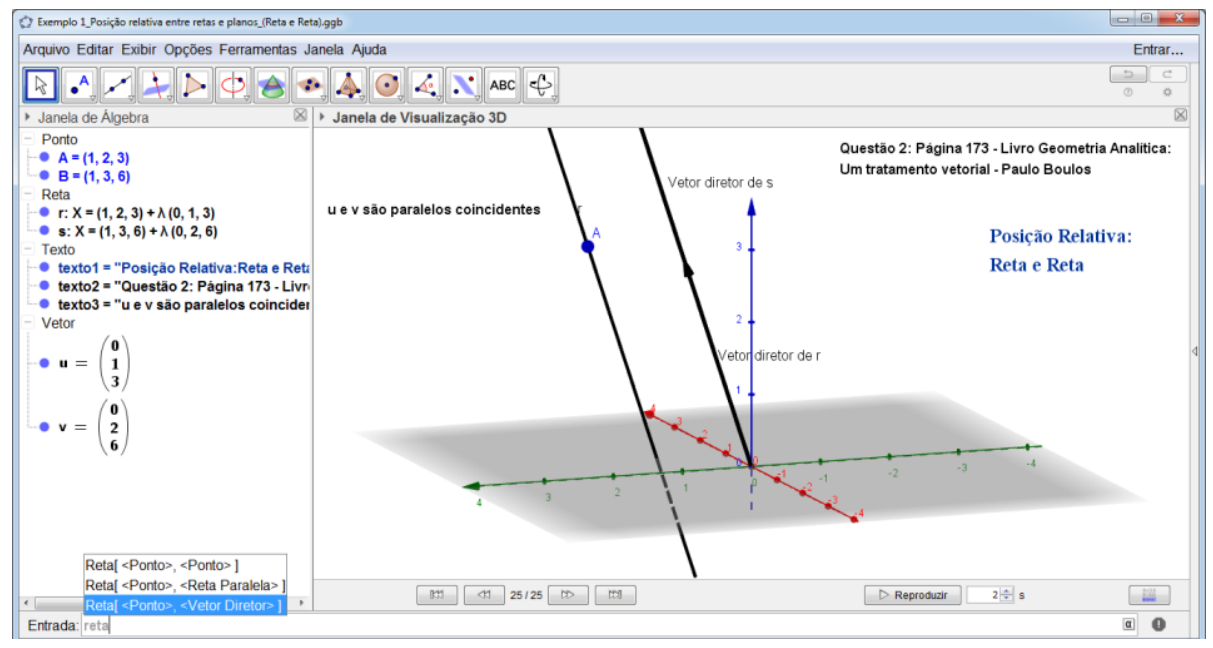




\section{Atividade 2 - Reta e Plano}

Esta segunda atividade é proposta em (BOULOS e CAMARGO, 2005), onde será verificado à posição relativa entre uma reta dada e um plano através de suas equações cartesianas. São dados o plano $\pi: 4 x+3 y-z-4$ e a reta $r: X=(1,1,1)+$ $\alpha(3,2,1)$. Estude a posição relativa de $r$ e $\pi$. Para abordar este problema de forma geométrica, seguiremos os mesmos procedimentos realizados no primeiro exemplo, digitando no campo de entrada à equação do plano $\pi$, denotado por $a$, na janela de álgebra do Geogebra, o ponto $A=(1,1,1)$, o vetor diretor da reta $r, \vec{v}=(3,2,1)$ e o vetor diretor normal ao plano $\pi$, dado por $\vec{u}=(4,3,-1)$. A resolução geométrica é mostrada na figura 4 , onde concluímos que a reta $r$ é transversal ao plano $\pi$, para mostrar esta transversalidade digitamos no campo de entrada o seguinte comando ângulo( $r, a)$, onde é calculado o ângulo entre a reta e o plano, cujo valor é $63^{\circ}$, o que caracteriza à posição transversal entre estes entes geométricos.

Figura 4: Interpretação geométrica da Atividade 2

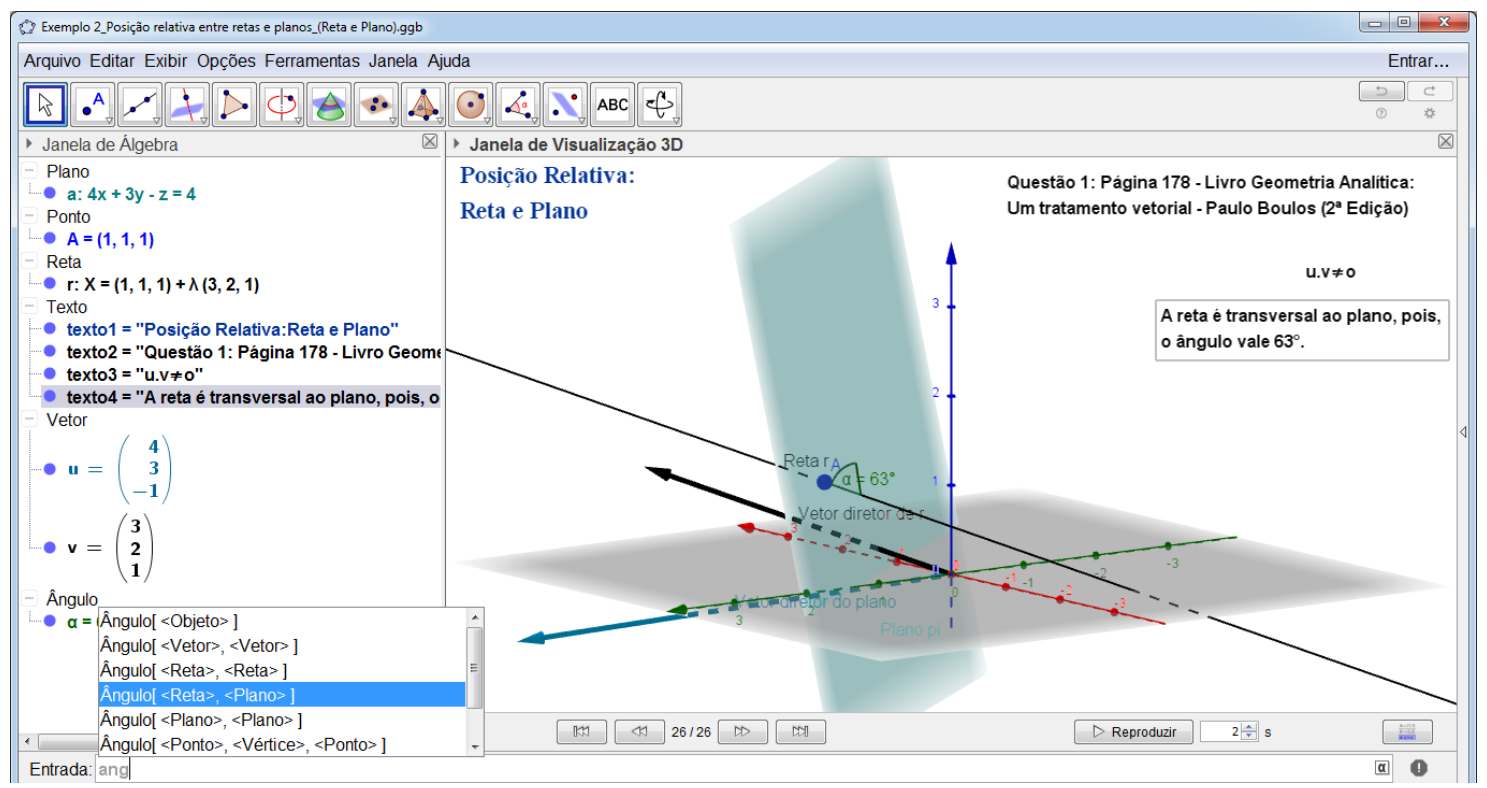

\section{Atividade 3 - Plano e Plano}

Na página 206 da referência supracitada, é proposto o seguinte problema: "Verificar se os planos $a: x+y-z-2=0 \quad$ e $\quad b: 4 x-2 y+2 z=0 \quad$ são perpendiculares".

Pelas equações cartesianas dos planos $a$ e $b$, podemos tirar seus respectivos vetores diretores normais $\vec{c}=(1,1,-1)$ e $\vec{u}=(4,-2,2)$ e digitá-los no campo de 
entrada do Geogebra. A solução geométrica é mostrada na figura 5, onde concluímos que os planos $a$ e $b$ são perpendiculares, verificando à inclinação entre eles, através do comando Ângulo(a,b), apertando a tecla ENTER, encontra-se o ângulo $\alpha=90^{\circ}$ o que comprova à perpendicularidade entre estes entes geométricos.

Figura 5: Interpretação geométrica da Atividade 3

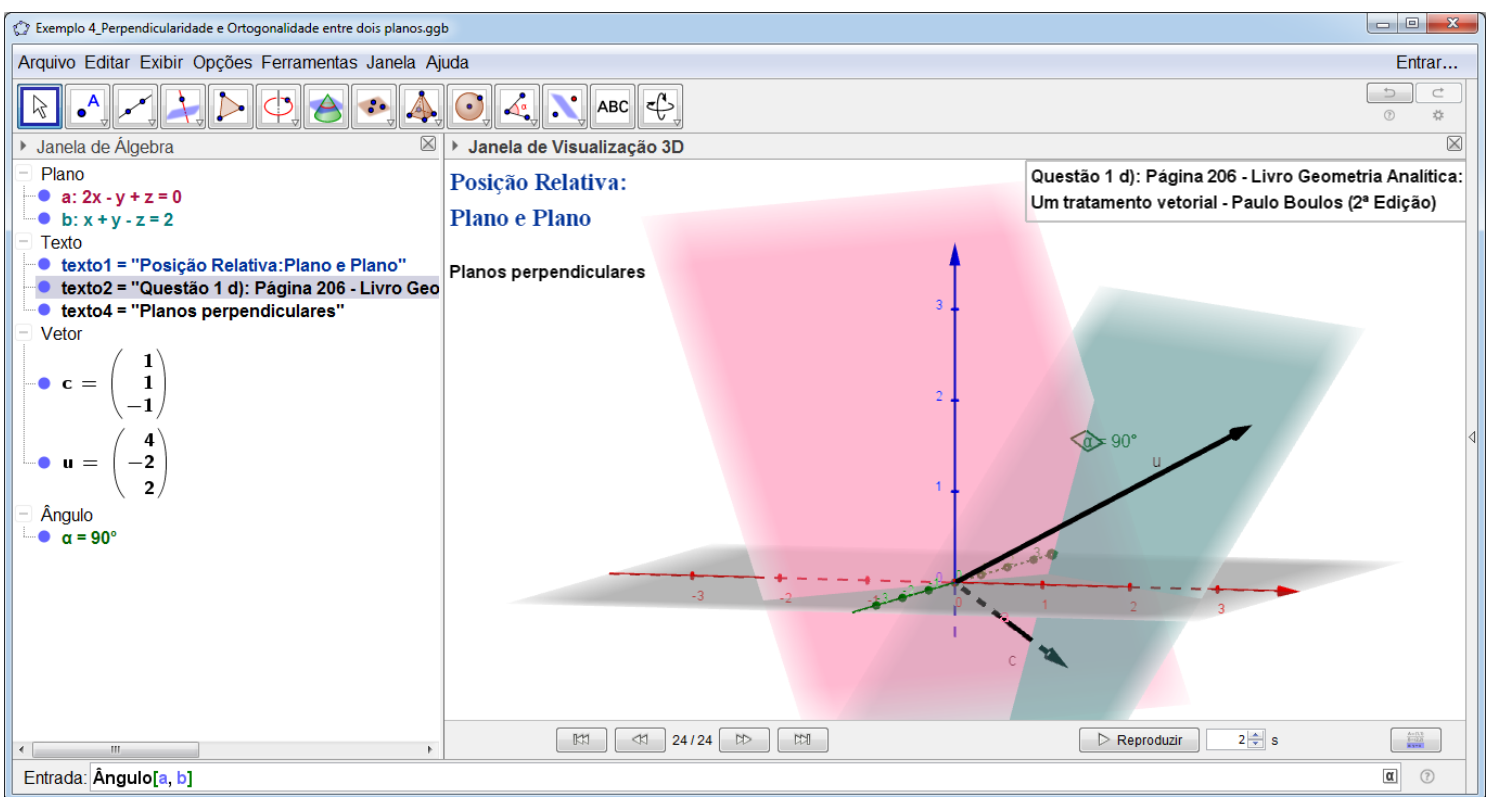

\section{Considerações finais}

Diante das análises e resultados auferidos pelos participantes desta pesquisa, vale ressaltar o importante relato dos alunos em relação às investigações realizadas no laboratório, onde os mesmos confirmam que a metodologia aplicada com o uso do Geogebra durante as simulações, onde elas, proporcionaram um bom desempenho no entendimento do conteúdo de Geometria Analítica sob uma nova perspectiva de observar à resolução dos problemas propostos, no que tange aos tópicos de posições relativas entre retas e planos, o que tornou este assunto mais interativo e acessível.

Este trabalho proporcionou à ampliação dos conhecimentos dos estudantes nas áreas em pesquisa como na Matemática, Geometria Analítica e Recursos Computacionais como ferramentas de investigação das aprendizagens correlatas. $\mathrm{O}$ avanço no conhecimento e aprendizado, se desencadeou, em virtude do desenvolvimento das novas metodologias aplicadas neste ramo da matemática, visando um completo entendimento do aluno, através das análises algébricas e geométricas destes conteúdos até então, vistos de maneira superficial, através de uma simples 
abordagem teórica, uma forma também de enriquecer a abordagem realizada na referência citada.

Assim, com os resultados alcançados pelos alunos, vemos que o papel do educador na arte de ensinar está se tornando cada vez mais desafiador com o passar dos tempos. Surgem cada vez mais novas possibilidades e oportunidades de se aplicarem recursos tecnológicos no ambiente de sala de aula para promover, sobretudo, um aprendizado interativo e significativo entre todos os envolvidos nas ações de ensinar e aprender. A partir dessas experiências propostas e realizadas, surgem reflexões que devem ser de todos os docentes da área, onde possam a cada prática educativa rever suas concepções de ensino e aprendizagem em que estes processos não podem ser resumidos a um mero processo de transmissão e aceitação de informações, e sim, pensados como um processo permanente de construção cognitiva que é estimulado pela investigação e participação dos discentes.

\section{Referências}

BORBA, M. C. Tecnologias Informáticas na Educação Matemática e Reorganização de Pensamento. In: BICUDO, M. A. V. (Org.). Pesquisa em Educação Matemática: concepções e perspectivas. São Paulo: UNESP, 1999.

BOULOS, P.;CAMARGO, I. Geometria Analítica - Um tratamento Vetorial. $3^{\text {a }}$ edição, São Paulo: Makron Books.

BRASIL. MEC. SEMT. Parâmetros Curriculares Nacionais: Ensino Médio. Brasília: Ministério da Educação, 2000.

D’Ambrosio, U.; BARros, J. P. D.. Computadores, Escola e Sociedade. São Paulo: Scipione, 1990.

GEOGEBRA-INFORMAÇÕES: Geogebra ajuda e busca: Universidade de Salzburg. Markus Hohenwarter. Traduzido por: Hermínio Borges Neto, Luciana de Lima, Alana Paula Araújo Freitas, Alana Souza de Oliveira. Disponível em: < https://app.geogebra.org/help/docupt_BR.pdf >. Acesso em: 02 de Setembro de 2016.

PONTE, J. P.; BROCARDO, J.; OLIVEIRA, H. Investigações Matemáticas na sala de aula. Belo Horizonte: Autêntica, 2003.

ZUlATtO, R. B. A. Professores de Matemática que utilizam Software de Geometria Dinâmica: suas características e perspectivas. Dissertação (Mestrado em Educação Matemática). Instituto de Geociências e Ciências Exatas. Universidade Estadual Paulista. Rio Claro, 2002. 